Supporting Information for:

\title{
Accelerating mineral carbonation using carbonic anhydrase
}

\author{
Ian M. Power*, Anna L. Harrison and Gregory M. Dipple
}

Department of Earth, Ocean and Atmospheric Sciences, The University of British Columbia, 2207 Main Mall, Vancouver, British Columbia V6T 1Z4, Canada.*IMP - ipower@eos.ubc.ca; ALH - aharriso@eos.ubc.ca; GMD-gdipple@eos.ubc.ca.

*Corresponding author.

Published in Environmental Science \& Technology

Number of pages: 14

Number of tables: 1

Number of figures: 7 


\section{Analytical methods}

\subsection{Characterization of brucite ore}

The chemical composition of the brucite used in experiments was determined using a Philips PW-1480 wavelength dispersive X-ray fluorescence (XRF) spectrometer. The surface area of the pulverized brucite was determined by multipoint BET with nitrogen gas adsorption using a Quantachrome Autosorb-1 surface area analyzer. Particle size distribution analysis was performed using a Malvern Mastersizer 2000 Laser Diffraction Particle Size Analyzer.

\subsection{X-ray diffraction methods}

Mineral phases in experimental precipitates were identified from X-ray diffraction (XRD) patterns with reference to the International Centre for Diffraction Database (ICDD) PDF-4+ 2010 using the program DIFFRACplus EVA $14 .{ }^{1}$ Finely ground aliquots were mounted as slurries onto glass plates with anhydrous ethanol and allowed to dry at room temperature. XRD data were collected using a Bruker D8 Focus Bragg-Brentano diffractometer with CoK $\alpha$ radiation. A long, fine-focus Co X-ray tube was operated at $35 \mathrm{kV}$ and $40 \mathrm{~mA}$ using a take-off angle of $6^{\circ}$. Data for mineral identification were collected with a step size of $0.04^{\circ} 2 \theta$ and counting time of $0.9 \mathrm{~s} / \mathrm{step}$ over a range of $3-80^{\circ} 2 \theta$ and a sample rotation of $50 \mathrm{rpm}$.

\subsection{Aqueous chemistry}

DIC concentrations were determined using an IL-550 TOC/TN analyzer with a calibration range of 5 to $500 \mathrm{mg} / \mathrm{L}$. Samples were dispensed into a sparge vessel and reacted with acid to release carbon as gaseous $\mathrm{CO}_{2}$, which was measured using an infrared detector. $\mathrm{Mg}$ concentrations were determined using inductively coupled plasma - optical emission 
spectrometry (ICP-OES) using a Varian 725-ES Optical Emission Spectrometer. Geochemical modelling of the experimental solutions using the PHREEQC code and LLNL database was performed to determine equilibrium $\mathrm{pH}$ and DIC concentrations as well as saturation indices of Mg-carbonate minerals. ${ }^{2}$

\subsection{Carbon abundance analysis}

The percent carbon (\% C) of the solid samples was determined using a Model CM501C4 $\mathrm{CO}_{2}$ Coulometer from UIC Inc. Calcium carbonate standards were analyzed to ensure instrument calibration. This method is accurate within $\pm 0.05 \%$ by mass with a detection limit of $0.10 \% \mathrm{C}$.

\subsection{Scanning electron microscopy}

Scanning electron microscopy (SEM) of solid samples was performed at the Centre for High-Throughput Phenogenomics at The University of British Columbia. Samples were coated with $5 \mathrm{~nm}$ of iridium using a Leica EM MED020 coating system and imaged using a FEI Helios NanoLab 650 operating at $1.0 \mathrm{kV}$ voltage. 


\section{PHREEQC model input file:}

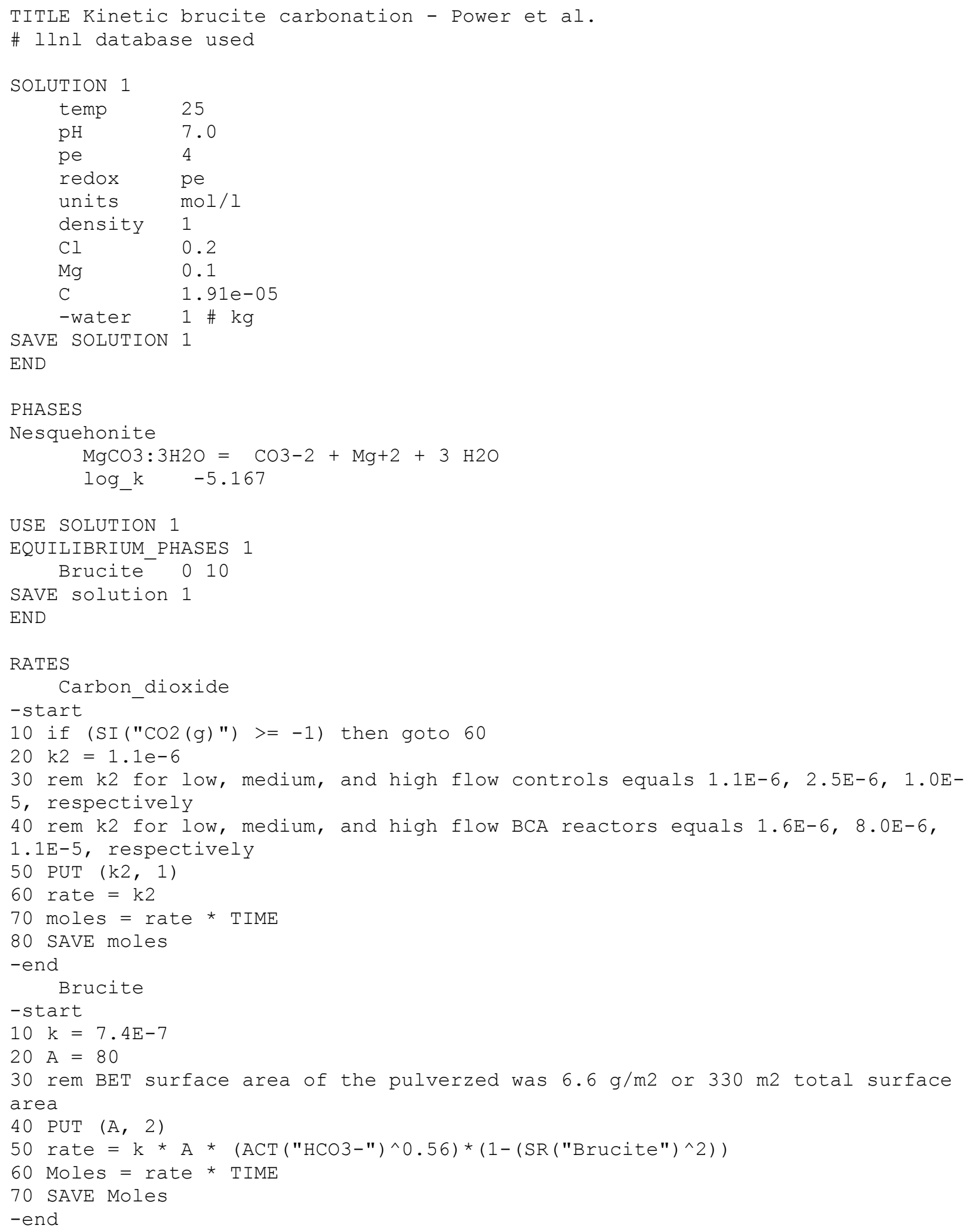




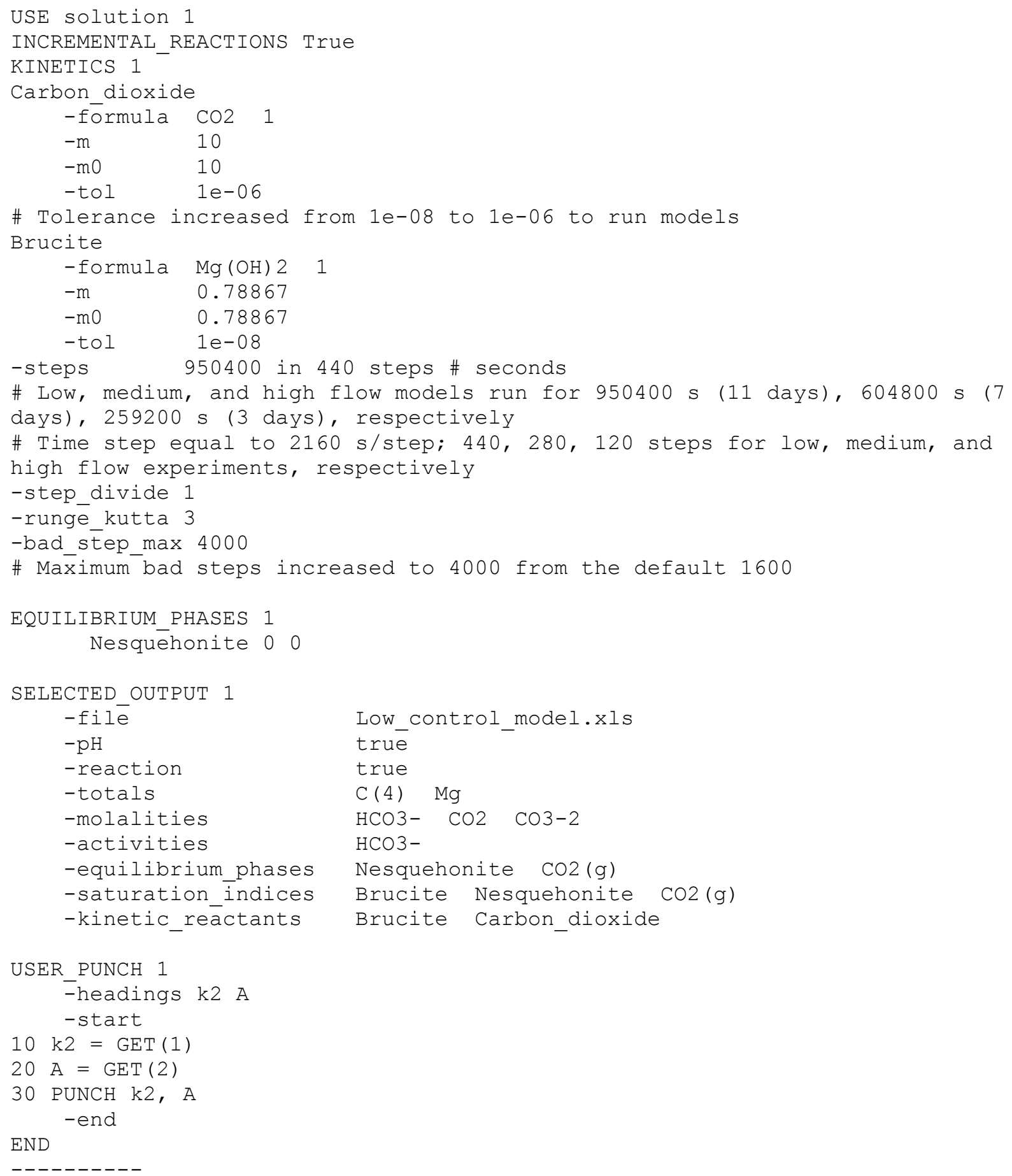




\begin{tabular}{cc}
\hline Oxide & Weight percent \\
\hline $\mathrm{SiO}_{2}$ & 1.74 \\
$\mathrm{TiO}_{2}$ & 0.01 \\
$\mathrm{Al}_{2} \mathrm{O}_{3}$ & 0.14 \\
$\mathrm{Fe}_{2} \mathrm{O}_{3}$ & 0.40 \\
$\mathrm{MnO}$ & 0.015 \\
$\mathrm{MgO}$ & 61.55 \\
$\mathrm{CaO}$ & 2.50 \\
$\mathrm{~K} 2 \mathrm{O}$ & $<0.01$ \\
$\mathrm{Na}_{2} \mathrm{O}$ & $<0.01$ \\
$\mathrm{P}_{2} \mathrm{O}_{5}$ & 0.01 \\
$\mathrm{Cr}_{2} \mathrm{O}_{3}$ & $<0.01$ \\
$\mathrm{L.O.I.}$ & 32.47 \\
Total & $\mathbf{9 8 . 8 3}$ \\
\hline Percent carbon & $\mathbf{1 . 1 0}$ \\
\hline Particle size $(\boldsymbol{\mu m})$ & $\mathbf{2 - 4 0}$ \\
\hline BET surface & \\
area $\left(\mathbf{m}^{2} / \mathbf{g}\right)$ & $\mathbf{6 . 6}$ \\
\hline
\end{tabular}

Table S1. Major oxides of the brucite ore used in experiments as determined by XRF analysis, and percent carbon, particle size, and BET surface area. ${ }^{3}$ 


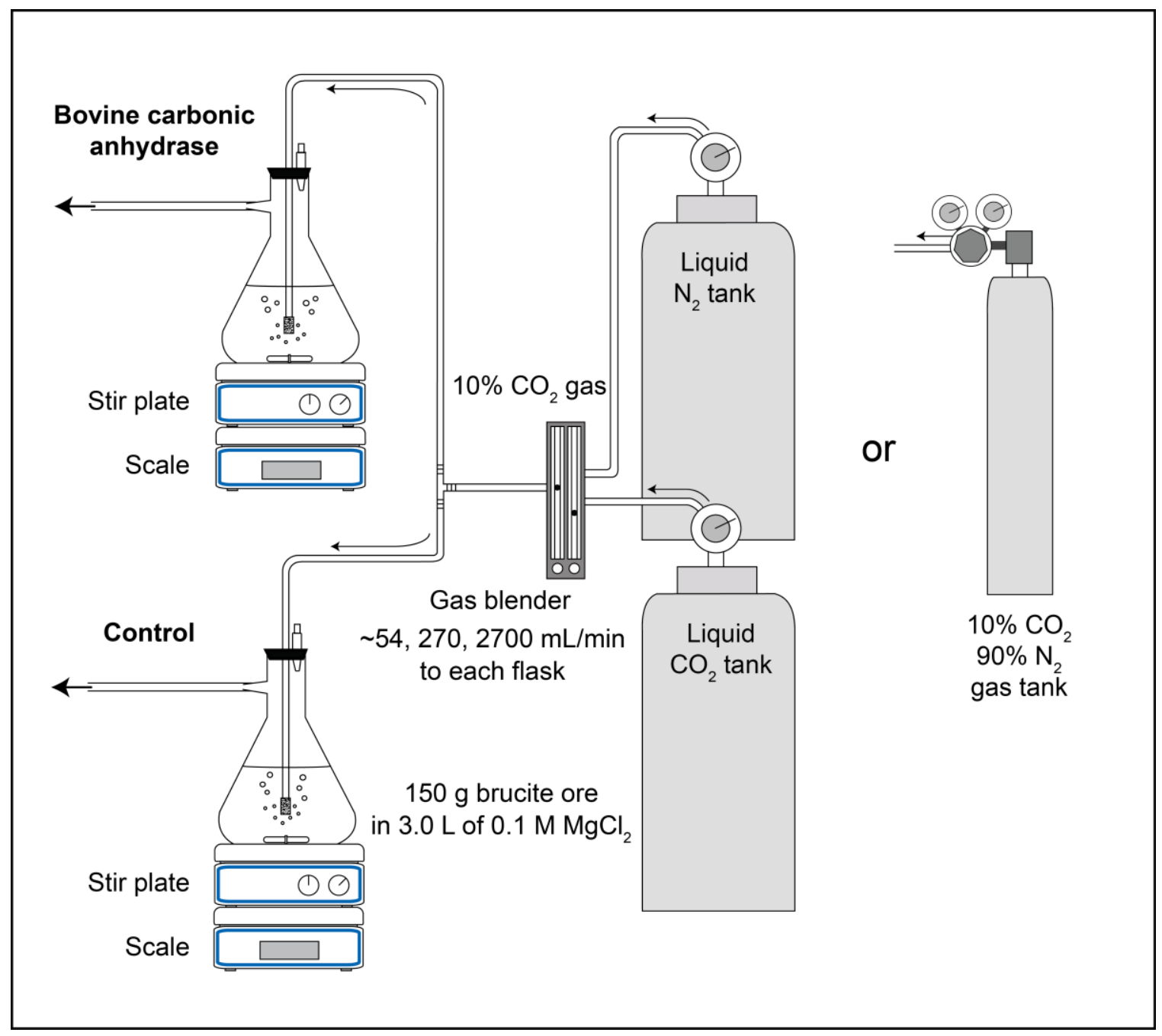

Figure S1. Schematic of brucite carbonation apparatus. 


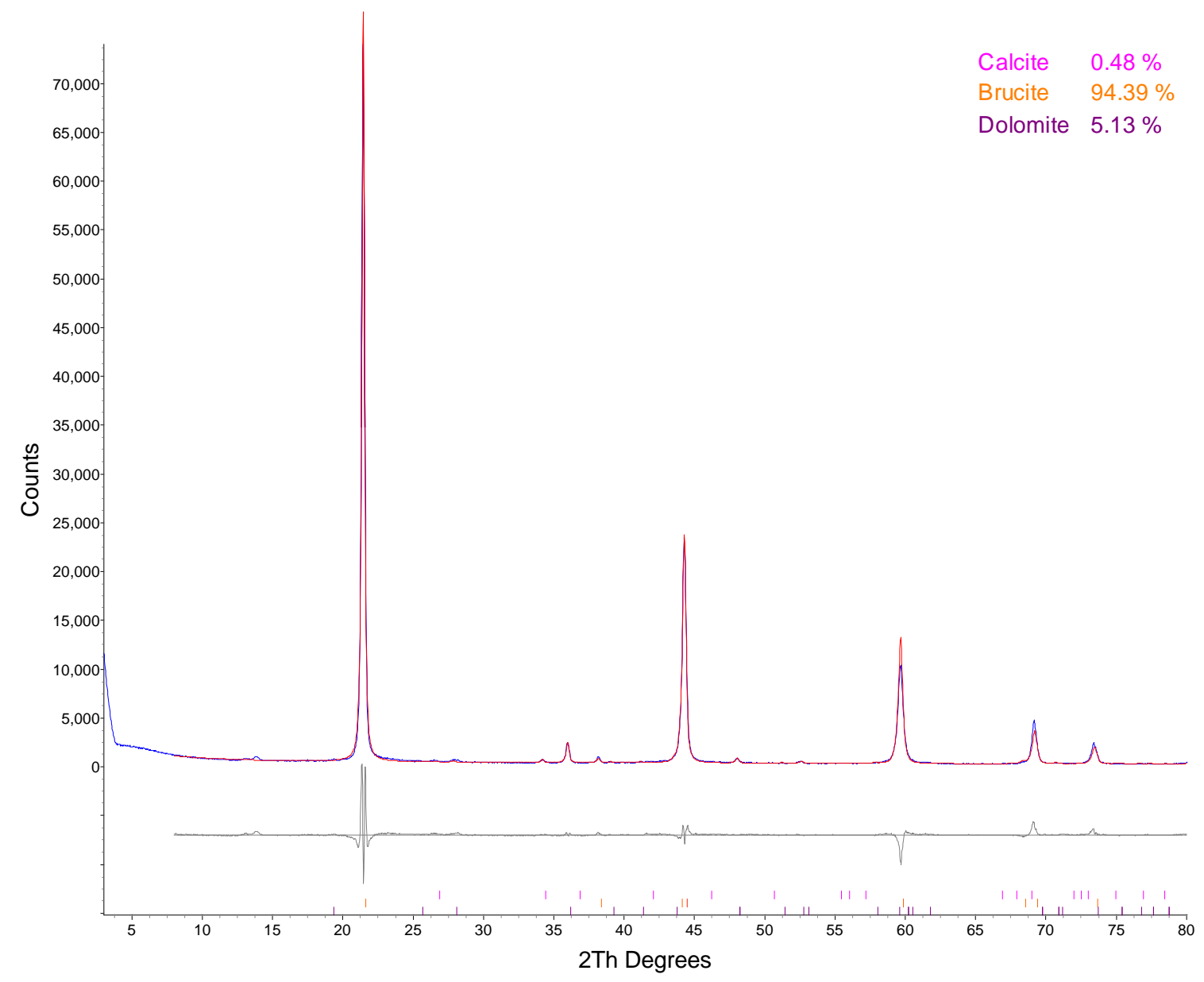

Figure S2. Rietveld refinement of XRD data from analysis of the intial brucite ore. 


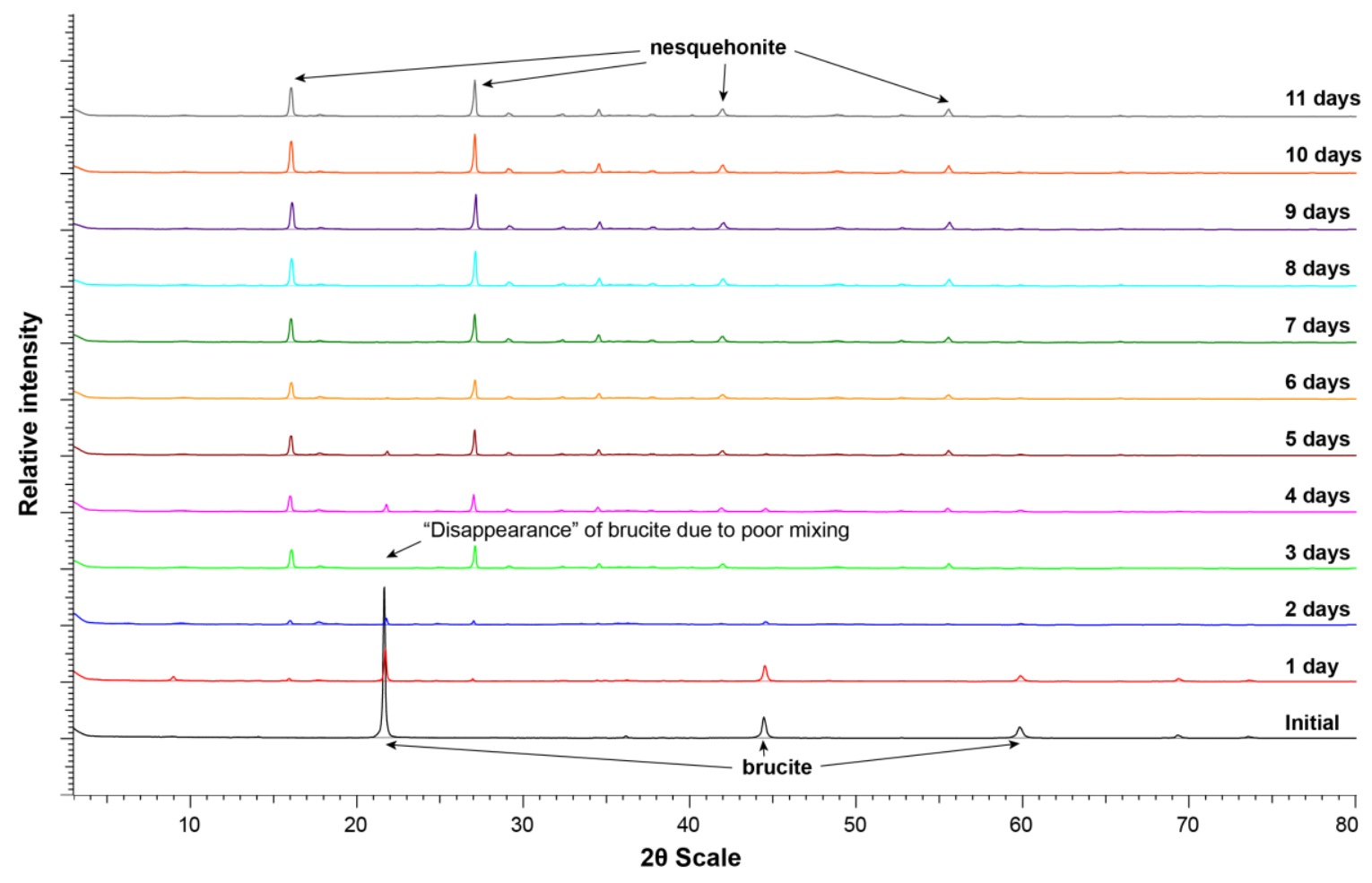

Figure S3. X-ray diffraction patterns of solids in the low flow control reactor with time. The most intense peaks of brucite and nesquehonite are indicated with arrows. 


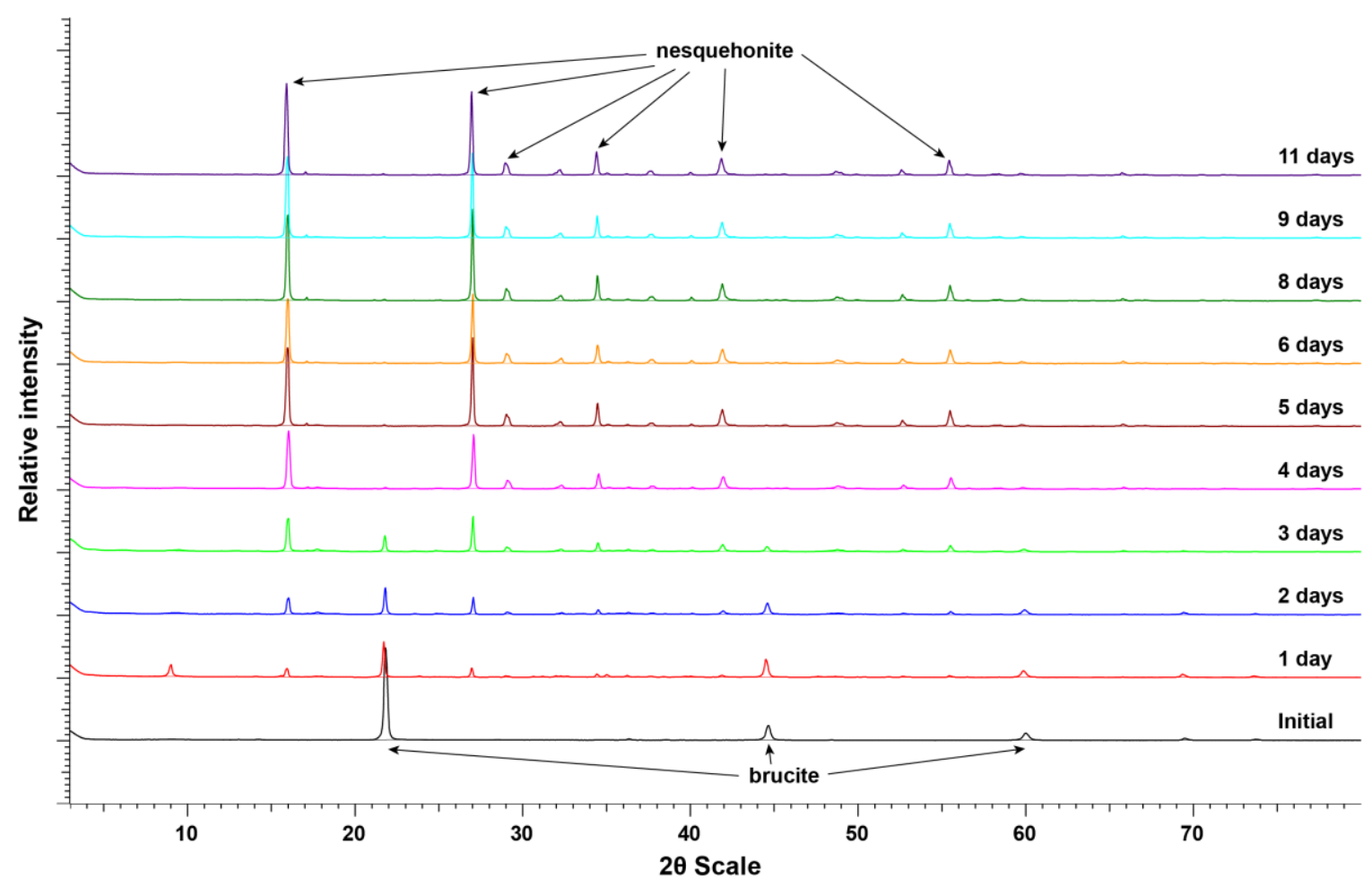

Figure S4. X-ray diffraction patterns of solids in the low flow BCA reactor with time. The most intense peaks of brucite and nesquehonite are indicated with arrows. 


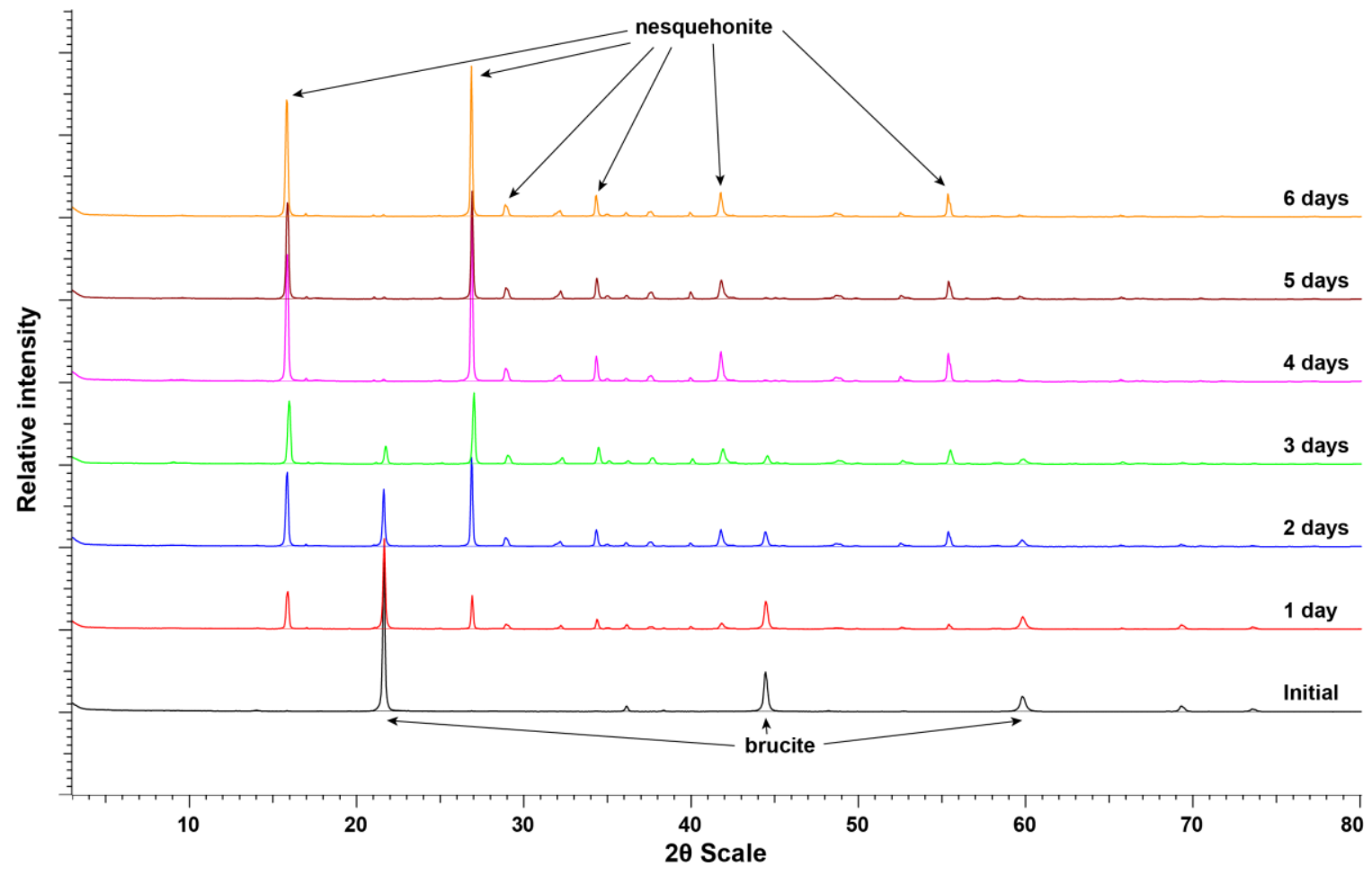

Figure S5. X-ray diffraction patterns of solids in the medium flow control reactor with time. The most intense peaks of brucite and nesquehonite are indicated with arrows. 


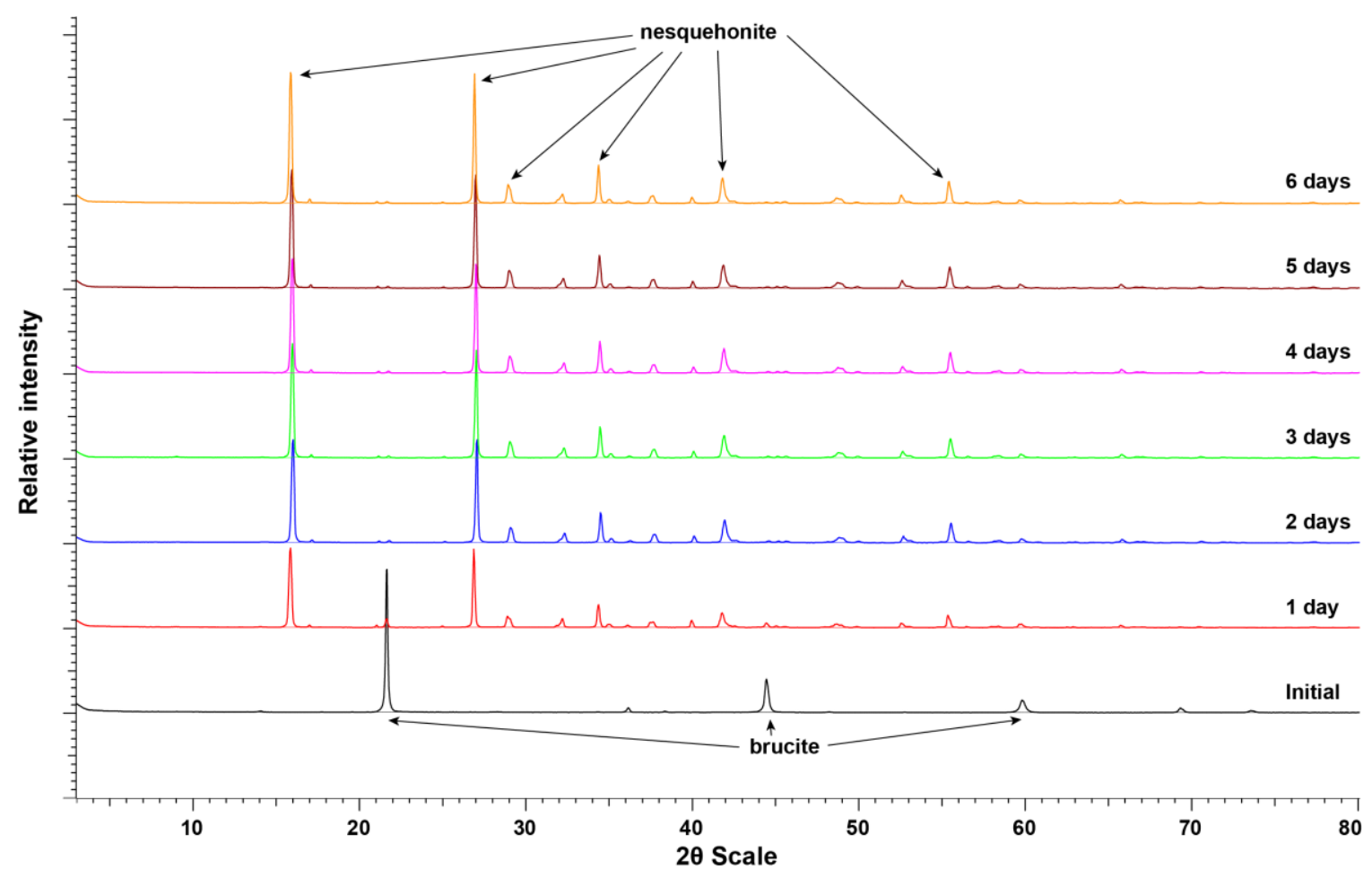

Figure S6. X-ray diffraction patterns of solids in the medium flow BCA reactor with time. The most intense peaks of brucite and nesquehonite are indicated with arrows. 


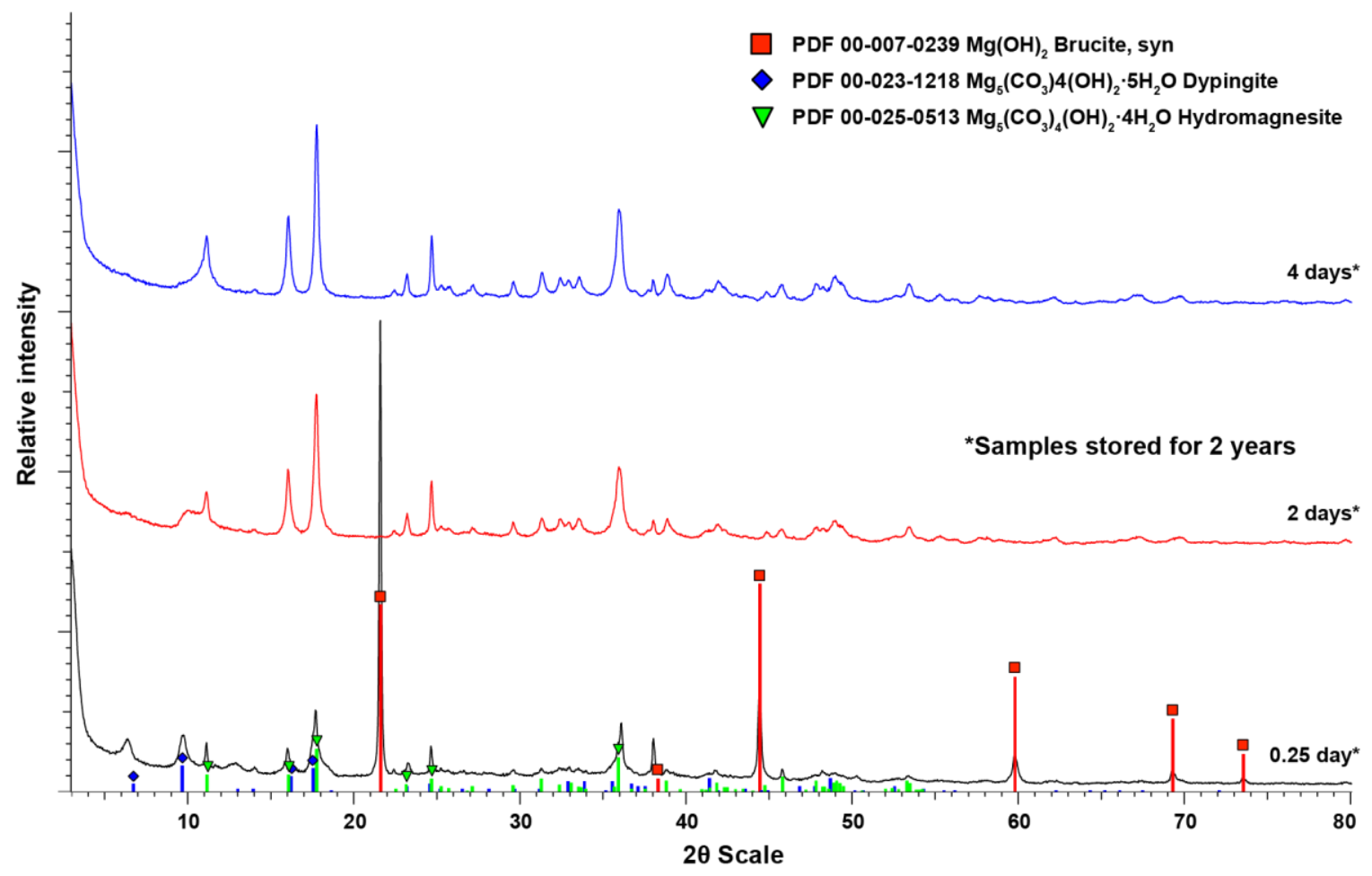

Figure S7. X-ray diffraction patterns of solids in the high flow BCA reactor with time. Solids were stored for two years prior to analysis with most carbonate having transformed to hydromagnesite. 


\section{Literature Cited}

1. Bruker AXS, DIFFRAC ${ }^{\text {plus }}$ EVA 14 Release 2008; Karlsruhe, Germany, 2008.

2. Parkhurst, D. L.; Appelo, C. A. J., Description of Input and Examples for PHREEQC Version 3 - A Computer Program for Speciation, Batch-Reaction, One-Dimensional Transport, and Inverse Geochemical Calculations. In U.S. Geological Survey Techniques and Methods, Denver, Colorado, 2013; Vol. book 6, p 497.

3. Power, I. M.; Harrison, A. L.; Dipple, G. M.; Southam, G., Carbon sequestration via carbonic anhydrase facilitated magnesium carbonate precipitation. International Journal of Greenhouse Gas Control 2013, 16, 145-155. 